\title{
Paper
}

\section{Statistical and Geometrical Methods: A Comparative Study on Color Transfer to Dark Image}

\author{
XIAOHUA ZHANG* Member, \\ YUELAN XIN ${ }^{\ddagger} \quad$ Non-member \\ Dongdong Cheng ${ }^{\dagger}$ Non-member
}

(Received February 29, 2020, revised April 19, 2020)

\begin{abstract}
Color transfer is a technical means of changing the appearance of an image (source image) such that the image has characteristics similar to those of a specified image (target image). There exist first-order statistics-based and geometry-based color transfer methods. These color transfer methods can be applied in different color spaces via space transformation. In this report, we review the fundamental principles of two color transfer methods in different color spaces and present a comprehensive comparative study on color transfer using statistical and geometrical methods in different color spaces. We assess the quality of color transfer by computing the consistency between the normalized histograms of the source image and target image and discuss the performance of each method for different color spaces. Our experiments show that the best color space is the RLAB color space while the geometrical method is quantitatively better than the statistical method for most of a large number of images.
\end{abstract}

Keywords: Color transfer, Color space, First-order statistics, Geometrical transformation, Histogram, Distance between histograms

\section{Introduction}

Color is an important feature of an image in the fields of painting and photography. Colors express the personal emotion and mood of the artist and present useful information for technical use such as in visualization. A common operation on an image is to alter the color in the image. Color transfer, also called color mapping, is a type of color manipulation that conveys the color information from a target image such that it has a color appearance similar to that of the target image. Figure 1 shows an example of color transfer, panel (a) is the source image, panel (b) is the target image, and panel (c) is the result of transferring the color information in panel (b) to panel (a) so that panel (c) has a color appearance similar to that of panel (b) while the contents of the scene remain unchanged. Color transfer is useful for color editing in image processing. As an example, color transfer is used to convey color information from a target image to a source image to change the appearance of the source image, such as from autumn to spring. It is also applied to reduce color differences between images in stitching a panorama using a series of images that may have slight color variations[1]. In the movie industry, consecutive shots taken at different times may have different colors due to slight changes in natural or artificial lighting,

\footnotetext{
* Hiroshima Institute of Technology, Hiroshima, Japan. (zhxh@cc.it-hiroshima.ac.jp)

$\dagger$ Yangtze Normal University, Chongqing, China.

(cdd@cqu.edu.cn)

* Qinghai Normal University, Xining, China.

(xinyue001112@163.com)
}

and this requires the color to be adjusted consistently across all shots[2]. In an image style transfer algorithm[3], when transferring the style in a given image to another image, the color distribution of the style image is also copied to the content image, which might be undesirable, and preservation of the color in the content image using a color transfer method may therefore be required[4].

In the graphics community, the pioneering work of color transfer was reported[5] by Reinhard et al. in 2001. It is well known that the three channels of the RGB color space have strong correlation. Reinhard et al. thus established a decorrelated color space $l \alpha \beta$ using a series of conversions from the RGB color space to the XYZ, to the LMS space, and finally to the $l \alpha \beta$ space. The decorrelation between the three color axes is computed through principal component analysis[5] [6]. Color is transferred on the decorrelated $l \alpha \beta$ color space and then converted back to the RGB color space. Xiao et al. proposed a method that transfers color from one image to another directly in the correlated RGB color space[7] without color space conversion. Both methods mentioned above transfer color globally (i.e., to the entire image); however, they mix up colors in different regions. In fact, these methods may be applied for corresponding parts of two images; i.e., swatch-based color transfer[5] [7]. An intensive review of color transfer, or color mapping, and its applications is presented in [1] [13]. Readers are strongly urged to find useful information in these references.

There are two predominant methods of color transfer. The method proposed by Reinhard et al.[5] computes the mean and standard deviations of all pixels along each of the 


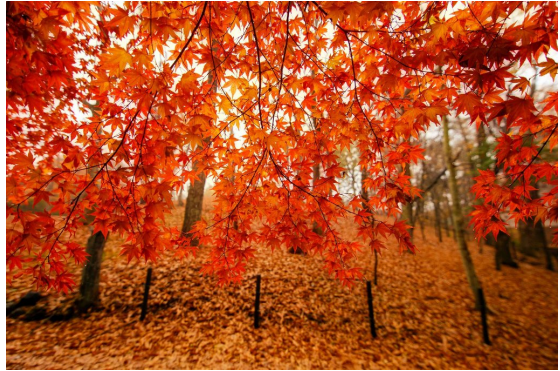

(a)

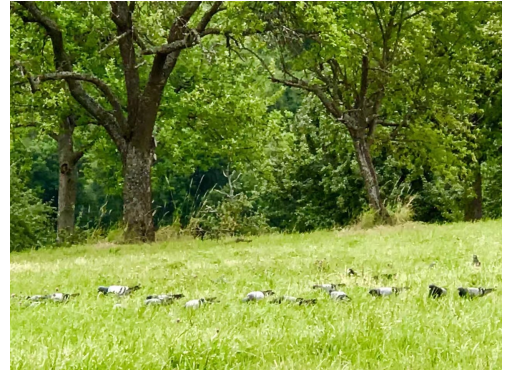

(b)

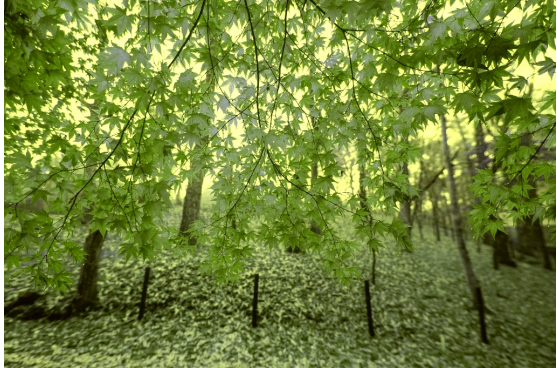

(c)

Figure 1: An example of color transfer: (a) source image, (b) target image, (c) result of transferring color information in (b) to (a) so that (c) has a color appearance similar to that of (b).

three axes in the $l \alpha \beta$ color space for target and source images. These statistical data are used to change the values of pixels in the source image in the $l \alpha \beta$ color space, and we thus refer to the method as the statistical method. Meanwhile, Xiao's method transforms the pixel distribution of the source image so that the image has a distribution similar to that of the target image. The method is essentially a geometrical transform using a series of translations, scalings, and rotations and we therefore refer to the method as the geometrical method.

Reinhard et al. used the $l \alpha \beta$ color space but their color transfer algorithm is not limited to this space. Many other related spaces, such as the $l a^{*} b^{*}, l u^{*} v^{*}$, and RLAB [8] color spaces, are also used in the literature. Jonathan et al. conducted an intensive series of experiments and claimed that the RLAB color space is the best space for transforming an image of a dark scene into an image of a lightened scene[9] using the statistical color transfer algorithm. Theoretically, the geometrical color transfer algorithm can also be applied in any color space and is not limited to the RGB color space.

In this paper, we conduct a comparative study of the statistical method [5] and geometrical method[7], which are the methods most commonly used in transferring color globally in various color spaces. We focus on five color spaces, namely the RGB, $l \alpha \beta, l a^{*} b^{*}, l u^{*} v^{*}$, and RLAB color spaces. The comparison uses distance metrics for histograms of the output image and target image in evaluating the quality of transferring color. We employ the four most commonly used distance metrics, namely, The Euclidean diastance, Bhattacharyya distance[10] [11], chi-square metric[12], and histogram intersection, to measure the similarity of histograms.

Section 2 describes the five typical color spaces used in the comparison and the conversions. The statistical and geometrical methods of color transfer between a source image and target image are detailed in Section 3. Section 4 presents experimental results and observations. Section 5 presents conclusions of the study and discusses future work.

\section{Color spaces}

Color transfer, or color mapping, is an operation that rearranges the pixel distribution of the source image in a color space, such that the distribution matches that of the target image. Many color spaces are adopted for color trans- fer algorithms. In our comparison, we apply the statistical method and geometrical method of color transfer in five commonly used color spaces, namely, the RGB, $l \alpha \beta, l a^{*} b^{*}$, and $l u^{*} v^{*}$, and a special RLAB color space. Conversions between the RGB color space and other spaces are described for completeness in this section.

2.1 RGB color space The first color space is the RGB space commonly used for image processing and computer graphics. The RGB color space is an additive color model that employs RGB primaries to define other colors by the three chromaticity values of red, green, and blue. In terms of digital image processing and computer graphics, the color model most commonly used in practice is the RGB model for a color monitor. In this model, each color appears in its primary spectral components as a triple of red, green, and blue. The model is based on a Cartesian coordinate system and the subspace of interest is a cube.

Theoretically, different colors representing a pixel in an image are points on or inside the cube in the model. It is often assumed that all colors are normalized so that the cube is the unit cube; i.e., all values $\mathrm{R}, \mathrm{G}$, and $\mathrm{B}$ are in the range $[0,1]$. In practice, an RGB image generally uses eight bits for each channel such that each color pixel has a depth of 24 bits, resulting in what is called a full-color or true color image[14].

2.2 $l \alpha \boldsymbol{\beta}$ color space As a non-standard color space, the $l \alpha \beta$ space was used by Reinhard et al. in their report[5]. Unlike the RGB color space, which has strong correlations among the three channels, the $l \alpha \beta$ color space has maximal decorrelation among the three axes computed in principal component analysis[15]. To compute $l \alpha \beta$ values from the RGB color space, we first convert the RGB color space to the XYZ space using the equation given by Reihard et al.[5]:

$$
\left(\begin{array}{l}
X \\
Y \\
X
\end{array}\right)=\left(\begin{array}{lll}
0.5141 & 0.3239 & 0.1604 \\
0.2651 & 0.6702 & 0.0641 \\
0.0241 & 0.1228 & 0.8444
\end{array}\right)\left(\begin{array}{c}
R \\
G \\
B
\end{array}\right) .
$$

This XYZ space is a device-independent color space. After converting the RGB space to the XYZ space, the next step 
is to convert the image to the LMS cone space:

$$
\left(\begin{array}{c}
L \\
M \\
S
\end{array}\right)=\left(\begin{array}{rrr}
0.3897 & 0.6890 & -0.0787 \\
-0.2298 & 1.1834 & 0.0464 \\
0.0000 & 0.0000 & 1.0000
\end{array}\right)\left(\begin{array}{c}
X \\
Y \\
Z
\end{array}\right) .
$$

Equations (1) and (2) can certainly be combined for conversion from the RGB space to the LMS space directly. Data distribution in the LMS space is heavily skewed, and thus converted to a logarithmic space to eliminate skewness. Data distribution in the logarithmic space is then converted to the decorrelated $l \alpha \beta$ color space:

$$
\left(\begin{array}{c}
l \\
\alpha \\
\beta
\end{array}\right)=\left(\begin{array}{ccc}
\frac{1}{\sqrt{3}} & 0 & 0 \\
0 & \frac{1}{\sqrt{6}} & 0 \\
0 & 0 & \frac{1}{\sqrt{2}}
\end{array}\right)\left(\begin{array}{rrr}
1 & 1 & 1 \\
1 & 1 & -2 \\
1 & -1 & 0
\end{array}\right)\left(\begin{array}{c}
\log L \\
\log M \\
\log S
\end{array}\right) .
$$

After transferring color in the decorrelated $l \alpha \beta$ color space, it is easy to convert the result image in the $l \alpha \beta$ color space to an image in the LMS space, XYZ space, and finally RGB space by a series of inverse conversions.

2.3 CIE $\boldsymbol{l a}^{*} \boldsymbol{b}^{*}$ color space The $l a^{*} b^{*}$ color space was designed by the International Commission on Illumination (CIE) in 1976 as a device-independent color model. In the $l a^{*} b^{*}$ color space, the magnitudes of numerical change in $l, a^{*}$, and $b^{*}$ correspond roughly to the same magnitudes of change visually perceived by human. The conversion between RGB and $l a^{*} b^{*}$ color spaces uses the deviceindependent CIEXYZ space converted from CIERGB and its reverse version. The forward transformation from the $\mathrm{XYZ}$ space to the $l a^{*} b^{*}$ space is

$$
\left\{\begin{array}{l}
l=116 f\left(\frac{Y}{Y_{n}}\right)-16 \\
a^{*}=500\left(f\left(\frac{X}{X_{n}}\right)-f\left(\frac{Y}{Y_{n}}\right)\right), \\
b^{*}=200\left(f\left(\frac{Y}{Y_{n}}\right)-f\left(\frac{Z}{Z_{n}}\right)\right)
\end{array}\right.
$$

where

$$
f(t)=\left\{\begin{array}{ll}
\sqrt[3]{t} & \text { if } t>\sigma^{3} \\
\frac{t}{3 \sigma^{2}}+\frac{4}{29} & \text { otherwise }
\end{array},\right.
$$

and $\sigma=6 / 29$. Note that $X_{n}, Y_{n}$, and $Z_{n}$ are a reference white point set to $(1.0,1.0,1.0)$ in this paper.

The reverse transformation from the $l a^{*} b^{*}$ space to the RGB space is simple and expressed as

$$
\left\{\begin{array}{l}
X=X_{n} f^{-1}\left(\frac{l+16}{116}+\frac{a^{*}}{500}\right) \\
Y=Y_{n} f^{-1}\left(\frac{l+16}{116}\right) \\
Z=Z_{n} f^{-1}\left(\frac{l+16}{116}-\frac{b^{*}}{200}\right)
\end{array},\right.
$$

where

$$
f^{-1}(t)=\left\{\begin{array}{ll}
t^{3} & \text { if } t>\sigma \\
3 \sigma^{2}\left(t-\frac{4}{29}\right) & \text { otherwise }
\end{array} .\right.
$$

The XYZ space is then converted to the RGB color space.
2.4 CIE $l \boldsymbol{u}^{*} \boldsymbol{v}^{*}$ color space The $l u^{*} v^{*}$ color space was designed by the CIE in 1976 as a simple transformation of the XYZ space. After converting from the CIERGB space to the CIEXYZ space, the conversion from the XYZ space to the $l u^{*} v^{*}$ space is as follows. Chromaticity coordinates $\left(u^{\prime}, v^{\prime}\right)$ are computed in the first step of conversion. Given a reference white point $\left(X_{n}, Y_{n}, Z_{n}\right)$, the coordinates $\left(u_{n}^{\prime}, v_{n}^{\prime}\right)$ are computed as

$$
\left\{\begin{array}{l}
u^{\prime}=\frac{4 X}{X+15 Y+3 Z} \\
v^{\prime}=\frac{9 Y}{X+15 Y+3 Z}
\end{array},\right.
$$

$l u^{*} v^{*}$ is then computed as

$$
\begin{cases}l=\left\{\begin{array}{cl}
(2 / \sigma)^{3} Y / Y_{n} & \text { if } Y / Y_{n} \leq \sigma^{3} \\
116\left(Y / Y_{n}\right)^{1 / 3}-16 & \text { otherwise }
\end{array}\right. \\
u^{*}=13 l \cdot\left(u^{\prime}-u_{n}^{\prime}\right) \\
v^{*}=13 l \cdot\left(v^{\prime}-v_{n}^{\prime}\right)\end{cases}
$$

where $\sigma=6 / 29$. The reverse transformation from $l u^{*} v^{*}$ to $\mathrm{XYZ}$ is

$$
\begin{aligned}
& \left\{\begin{array}{l}
u^{\prime}=\frac{u^{*}}{13 l}+u_{n}^{\prime} \\
v^{\prime}=\frac{v^{*}}{13 l}+v_{n}^{\prime}
\end{array}\right. \\
& \left\{\begin{array}{l}
Y=\left\{\begin{array}{ll}
Y_{n} l(\sigma / 2)^{3} & \text { if } l \leq 8 \\
Y_{n}\left(\frac{l+16}{116}\right)^{3} & \text { otherwise } \\
X & =Y\left(\frac{9 u^{\prime}}{4 v^{\prime}}\right) \\
Z & =Y\left(\frac{12-3 u^{\prime}-20 v^{\prime}}{4 v^{\prime}}\right)
\end{array} .\right.
\end{array}\right.
\end{aligned}
$$

Again, it is easy to convert the XYZ space back to the RGB color space.

2.5 RLAB color space The RLAB color space was originally designed for fixing problems with the CIE $l a^{*} b^{*}$ color space that arise under unusual lighting conditions[8]. In the conversion between the RGB and RLAB color spaces, the CIEXYZ space is converted to an XYZ space under reference viewing conditions:

$$
\left[\begin{array}{c}
X_{\text {ref }} \\
Y_{\text {ref }} \\
Z_{\text {ref }}
\end{array}\right]=\mathbf{R A M}\left[\begin{array}{c}
X \\
Y \\
Z
\end{array}\right]
$$

where

$$
\mathbf{R A M}=\left(\begin{array}{rrr}
1.0020 & -0.0401 & 0.0084 \\
-0.0042 & 0.9666 & 0.0008 \\
0.0000 & 0.0000 & 0.9110
\end{array}\right) .
$$




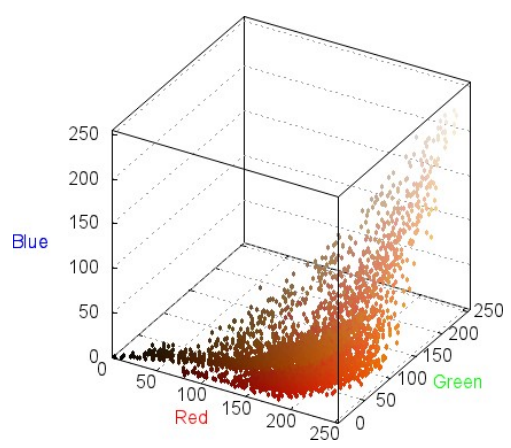

(a)

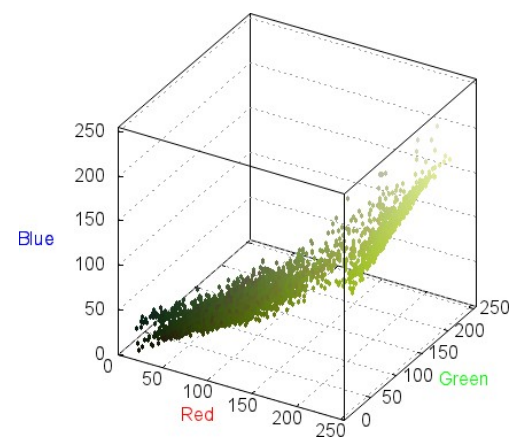

(b)

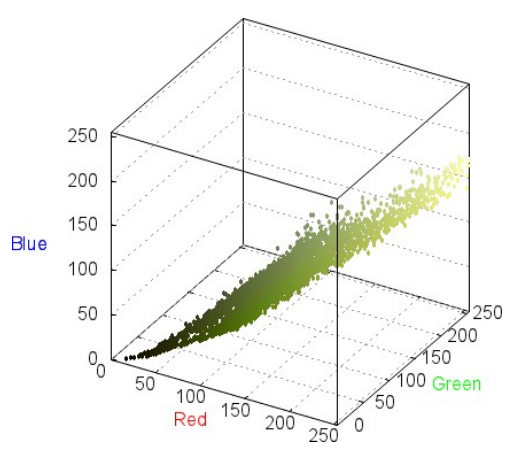

(c)

Figure 2: Pixel distributions: (a), (b), and (c) are distributions of pixels sampled from images in Fig. 1(a), (b), and (c) in the RGB color space.

In the forward transformation, the three components $(l, a, b)$ in the RLAB color space are computed as

$$
\left\{\begin{array}{l}
l=100 Y_{r e f}^{\sigma} \\
a=430\left(X_{r e f}^{\sigma}-Y_{r e f}^{\sigma}\right) \\
b=170\left(Y_{r e f}^{\sigma}-Z_{r e f}^{\sigma}\right)
\end{array}\right.
$$

where the exponent $\sigma$ takes the value of $1 / 2.3$ for an average-brightness surround, $1 / 2.9$ for a dim surround, and $1 / 3.5$ for a dark surround as suggested in [8]. The backward transformation from the RLAB space back to the $\mathrm{XYZ}$ is trivial:

$$
\begin{aligned}
& \left\{\begin{array}{l}
Y_{r e f}=\left(\frac{l}{100}\right)^{1 / \sigma} \\
X_{r e f}=\left(\frac{a}{430}+Y_{r e f}^{\sigma}\right)^{1 / \sigma} \\
Z_{r e f}=\left(Y_{r e f}^{\sigma}-\frac{b}{170}\right)^{1 / \sigma}
\end{array}\right. \\
& {\left[\begin{array}{c}
X \\
Y \\
Z
\end{array}\right]=\mathbf{R A M}^{-1}\left[\begin{array}{c}
X_{r e f} \\
Y_{r e f} \\
Z_{r e f}
\end{array}\right] .}
\end{aligned}
$$

The XYZ space is then converted back to the RGB color space.

\section{Color transfer}

The operations of color transference are conducted in one of the above five color spaces. Without loss of generality, we refer to any color space as $C$ and the three channels of the color space as $C_{k}, k=1,2,3$. The statistical and geometrical color transfer methods are considered for comparative study and are briefly described in this section.

3.1 Statistical method Reinhard et al. proposed a statistical method of color transfer[5]. They used first-order statistics; i.e., the global mean and standard deviation of both source and target images. The method modifies the content of the source image using statistics from the target image. This method was originally used in the $l \alpha \beta$ decorrelated color space but is not limited to this space. We apply the method in other color spaces for a comparison of performance. Given a color space $C$ with three channels $C_{k}, k=1,2,3$, the first statistics are computed for each channel and for both the source and target images. Supposing that the image size is $M_{s} \times N_{s}$ for the source image $S$ and $M_{t} \times N_{t}$ for the target image $T$, the means and standard deviations are computed as

$$
\begin{aligned}
& \left\{\begin{array}{l}
\mu_{k}^{s}=\frac{1}{M_{s} N_{s}} \sum_{x=0}^{M_{s}-1} \sum_{y=0}^{N_{s}-1} S_{k}(x, y) \\
\mu_{k}^{t}=\frac{1}{M_{t} N_{t}} \sum_{x=0}^{M_{t}-1} \sum_{y=0}^{N_{t}-1} T_{k}(x, y)
\end{array}\right. \\
& \left\{\begin{array}{l}
\sigma_{k}^{s}=\sqrt{\frac{1}{M_{s} N_{s}} \sum_{x=0}^{M_{s}-1} \sum_{y=0}^{N_{s}-1}\left(S_{k}(x, y)-\mu_{k}^{s}\right)^{2}} \\
\sigma_{k}^{t}=\sqrt{\frac{1}{M_{t} N_{t}} \sum_{x=0}^{M_{t}-1} \sum_{y=0}^{N_{t}-1}\left(T_{k}(x, y)-\mu_{k}^{t}\right)^{2}}
\end{array}\right.
\end{aligned}
$$

where $\mu$ and $\sigma$ are respectively the mean and standard deviation, $s$ and $t$ respectively represent the source image and target image, $k$ is the channel index, and $S_{k}(x, y)$ and $T_{k}(x, y)$ respectively indicate the source and target pixel values at $(x, y)$ in channel $k$ of a given color space, which is the RGB, $l \alpha \beta, l a^{*} b^{*}, l u^{*} v^{*}$, or RLAB color space.

The color transfer from the target image $T$ to the source image for channel $k$ is implemented by subtraction, scaling, and addition operations. This statistical color transfer is simple and intuitive and can be implemented adopting

$$
D_{k}(x, y)=\frac{\sigma_{k}^{t}}{\sigma_{k}^{s}}\left(S_{k}(x, y)-\mu_{k}^{s}\right)+\mu_{k}^{t},
$$

where $D_{k}(x, y)$ represents the destination image for channel $k$ and pixel $(x, y)$ after color transfer. All channels are processed the same way.

3.2 Geometrical method The geometrical method of color transfer focuses on the distribution of pixels in a given 


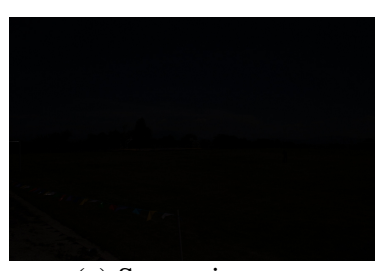

(a) Source image

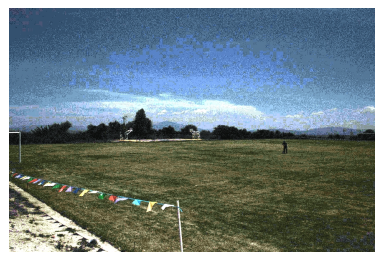

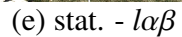

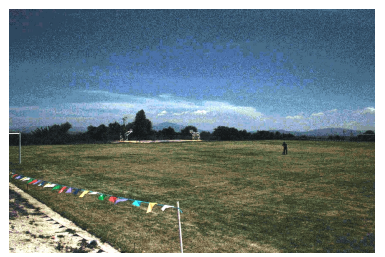

(i) stat. $-l u^{*} v^{*}$

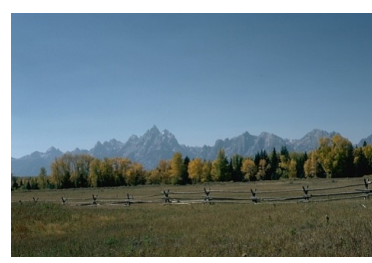

(b) Target image

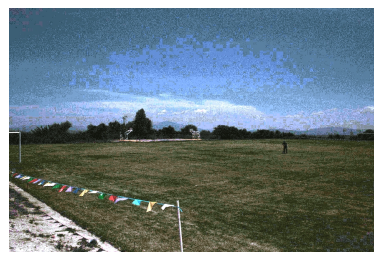

(f) geom. - l $\alpha \beta$

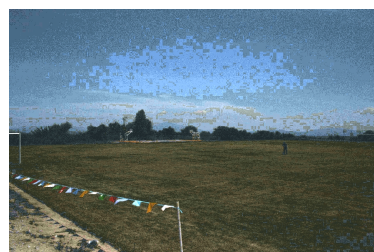

(j) geom. $-l u^{*} v^{*}$

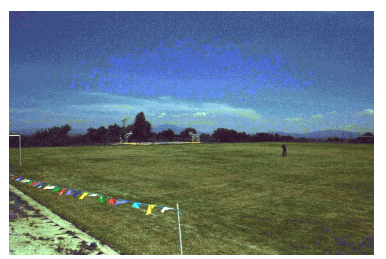

(c) stat. - RGB

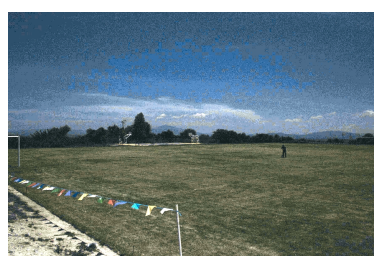

(g) stat. - $l a^{*} b^{*}$

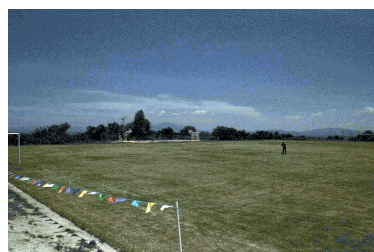

(k) stat. - RLAB

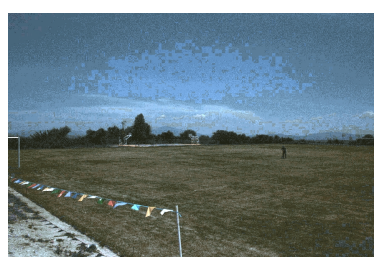

(d) geom. - RGB

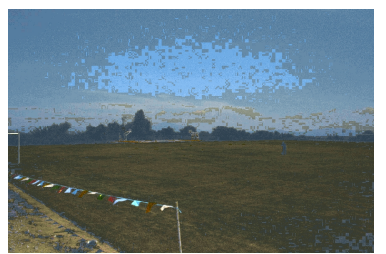

(h) geom. - $l a^{*} b^{*}$

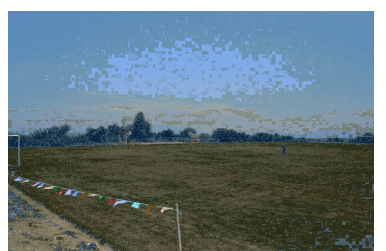

(1) geom. - RLAB

Figure 3: Example of color transfer using natural dark images: (a) source image, (b) target image, (c)-(1) results obtained using the statistical and geometrical methods in the order of RGB, $l \alpha \beta, l a^{*} b^{*}, l u^{*} v^{*}$, and RLAB color spaces. Note that stat. $=$ statistical method, geom. $=$ geometrical method.

Table 1: Quantitative evaluation of the images in Fig. 3 by histogram matching

\begin{tabular}{|l|cc|cc|cc|cc|}
\hline \multirow{2}{*}{ Color space } & \multicolumn{2}{|c|}{ Euclidean } & \multicolumn{2}{|c|}{ Bhattacharyya } & \multicolumn{2}{c|}{ Chi-square } & \multicolumn{2}{c|}{ Intersection } \\
\cline { 2 - 9 } & stat. & geom. & stat. & geom. & stat. & geom. & stat. & geom. \\
\hline RGB & 0.7689 & 0.4517 & 1.5542 & 1.3237 & 3.1879 & 2.5314 & 0.0746 & 0.3099 \\
\hline$l \alpha \beta$ & 0.5173 & 0.4923 & 1.4241 & 1.4187 & 2.7918 & 2.7925 & 0.2163 & 0.2167 \\
\hline$l a^{*} b^{*}$ & 0.4655 & 0.4499 & 1.3699 & 1.2855 & 2.6348 & 2.4338 & 0.2696 & 0.3379 \\
\hline$l u^{*} v^{*}$ & 0.4797 & 0.4530 & 1.4115 & 1.3361 & 2.7691 & 2.5840 & 0.2188 & 0.2798 \\
\hline RLAB & 0.4674 & 0.4548 & 1.3374 & 1.3111 & 2.5658 & 2.5113 & 0.2970 & 0.3157 \\
\hline
\end{tabular}

The Italic number in a numerical cell indicates the best value of a metric. stat. = statistical method; geom. $=$ geometrical method.

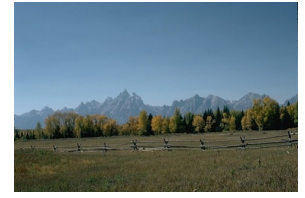

(a)

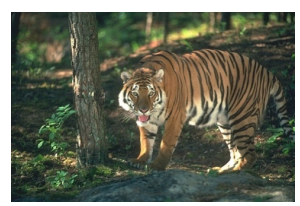

(f)

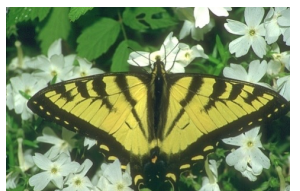

(b)

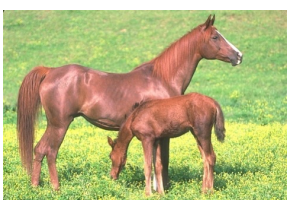

(g)

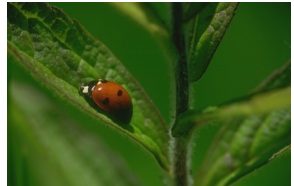

(c)

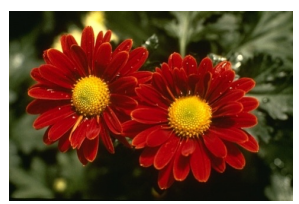

(h)

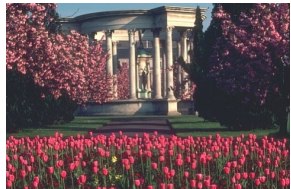

(d)

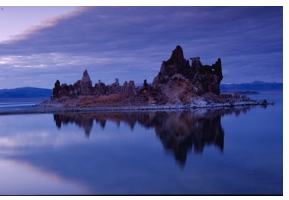

(i)

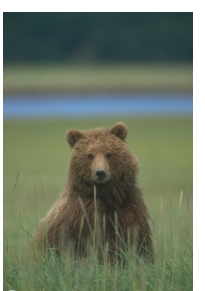

(e)

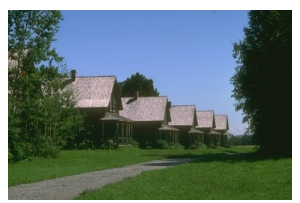

(j)

Figure 4: Ten images from the BSD300 dataset used as target images for color transfer.

color space[7] [16]. The method rearranges the pixel distribution of a source image so that it has a distribution sim- ilar to that of a target image. This rearrangement of pixel distributions comprises a series of geometrical transforma- 
Table 2: Averages of the quantitative evaluation of all dark images by histogram matching

\begin{tabular}{|l|cc|cc|cc|cc|}
\hline \multirow{2}{*}{ Color space } & \multicolumn{2}{|c|}{ Euclidean } & \multicolumn{2}{|c|}{ Bhattacharyya } & \multicolumn{2}{c|}{ Chi-square } & \multicolumn{2}{c|}{ Intersection } \\
\cline { 2 - 9 } & stat. & geom. & stat. & geom. & stat. & geom. & stat. & geom. \\
\hline RGB & 0.6434 & 0.3951 & 1.4469 & 1.0443 & 2.8244 & 1.7769 & 0.2241 & 0.6336 \\
\hline$l \alpha \beta$ & 0.4005 & 0.3863 & 1.0870 & 1.0580 & 1.8846 & 1.8125 & 0.5899 & 0.6158 \\
\hline$l a^{*} b^{*}$ & 0.4558 & 0.4109 & 1.1326 & 1.0672 & 2.0016 & 1.8412 & 0.5457 & 0.6017 \\
\hline$l u^{*} v^{*}$ & 0.4152 & 0.3862 & 1.0961 & $\mathbf{1 . 0 3 4 8}$ & 1.9111 & 1.7661 & 0.5748 & 0.6319 \\
\hline RLAB & $\mathbf{0 . 3 9 4 1}$ & $\mathbf{0 . 3 8 2 3}$ & $\mathbf{1 . 0 8 2 7}$ & 1.0371 & $\mathbf{1 . 8 6 5 2}$ & $\mathbf{1 . 7 5 5 8}$ & $\mathbf{0 . 5 9 6 8}$ & $\mathbf{0 . 6 3 6 7}$ \\
\hline
\end{tabular}

The Italic number in a numerical cell indicates the best value of a metric.

The bold number is the best value in a column.

stat. = statistical method; geom. = geometrical method.

tions, such as translations, rotations, and scalings. Supposing the transformation is represented by a matrix $\mathcal{M}$, the color transfer operation is then written as $D=\mathcal{M S}$ for each pixel, where $D=\left(D_{1}, D_{2}, D_{3}\right)^{T}$ and $S=\left(S_{1}, S_{2}, S_{3}\right)^{T}$ represent pixel values for the three channels of the destination and source images, with ${ }^{T}$ indicating the matrix transpose operation.

The transformation matrix $\mathcal{M}$ transforms comprise of a series of translations, rotations, and scalings. The matrix is written as

$$
\begin{aligned}
\mathcal{M} & =\mathcal{M}_{t} \cdot \mathcal{M}_{s} \\
& =\mathcal{T}_{t} \cdot \mathcal{R}_{t} \cdot \mathcal{S}_{t} \cdot \mathcal{S}_{s} \cdot \mathcal{R}_{s} \cdot \mathcal{T}_{s},
\end{aligned}
$$

where the matrix $\mathcal{T}_{\{t, s\}}$ represents translations between the center of the distribution and the origin of the coordinate system, $\mathcal{R}_{\{t, s\}}$ represents rotations between the principal axes and the coordinate axes, and $\mathcal{S}_{\{t, s\}}$ represents the scalings between unit scales and distribution scales. The matrix $\mathcal{M}_{s}$ transforms the pixel distribution of the source image to a normalized distribution, where the matrix $\mathcal{T}_{s}$ translates pixels of the source image so that the center of the distribution is at the origin, $\mathcal{R}_{s}$ rotates pixels so that the principal axes are in accordance with the axes of the coordinate system, and $\mathcal{S}_{s}$ scales pixels to the unit scale. The matrix $\mathcal{M}_{t}$ acts in a reverse manner by transforming the normalized distribution so that the distribution is similar to that of the target image. The matrix $\mathcal{S}_{t}$ scales the unit scale to that of the distribution of the target image, $\mathcal{R}_{t}$ rotates the axes of the coordinate system to the principal axes of the distribution of the target image, and $\mathcal{T}_{t}$ translates the origin to the center of the distribution of the target image. The translation matrices $\mathcal{T}_{\{t, s\}}$ are computed by finding the centroids of distributions of both source and target images. The rotation $\mathcal{R}_{\{t, s\}}$ and scaling $\mathcal{S}_{\{t, s\}}$ matrices are calculated from covariance matrices of source and target images, using SVD decomposition[17]. Details are found in [7] [16].

Figure 2 presents an example to explain the effect of color transfer using the geometrical method. Figure 2(a), (b), and (c) shows distributions of pixels sampled from images in Fig. 1(a), (b), and (c), respectively, in the RGB color space. It is seen that the source and target images have very different distributions; however, after transferring the color information in the target image to the source image, the resulting image has a distribution very similar to that of the target image.

\section{Quantitative evaluation}

The color transfer operation maps color information from the target image to the source image. The resulting image should have color appearance similar to that of the target image. A method of measuring the similarity of the appearances of the images is to adopt a histogram similarity metric. Suppose a histogram has $b$ bins. A histogram can then be considered as a point in a $b$-dimensional space. A histogram similarity metric is in fact the distance metric between the two points representing the two histograms. This metric has been used in evaluating image similarity[18] previously.

Although there are many metrics, in our comparative study, we used four metrics to compute how the histograms match, namely the Euclidean distance $d_{E}^{k}$, Bhattacharyya distance $d_{B}^{k}$, chi-square metric $d_{C}^{k}$, and intersection $d_{I}^{k}$ for channel $k$. In the following metric calculations, $H_{d}^{k}$ and $H_{t}^{k}$ represent the histograms of result and target images, and all histograms have bins of $b=256$ for each channel. Moreover, these histograms are normalized so that each histogram has a unit sum of $b=256$ bins; i.e., $\sum_{i=0}^{b-1} H(i)=1.0$.

The Euclidean distance between two histograms $H_{d}^{k}$ and $H_{t}^{k}$ is defined as the distance between two points in $b$-dimensional space:

$$
d_{E}^{k}\left(H_{d}^{k}, H_{t}^{k}\right)=\sqrt{\sum_{i=0}^{b-1}\left(H_{d}^{k}(i)-H_{t}^{k}(i)\right)^{2}},
$$

where the Euclidean distance $d_{E}^{k}$ is in the range $[0.0, \sqrt{2}]$.

A histogram is considered a probability distribution of pixel values. The Bhattacharyya distance measures the similarity of two distributions. Note that the histograms have been normalized. We have

$$
d_{B}^{k}\left(H_{d}^{k}, H_{t}^{k}\right)=\sqrt{1-\sum_{i=0}^{b-1} \sqrt{\left(H_{d}^{k}(i) \cdot H_{t}^{k}(i)\right)}}
$$

where the Bhattacharyya distance is in the range $[0.0,1.0]$.

There are several definitions for the chi-square metric. 


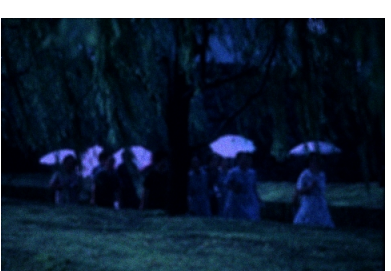

(a) Source image

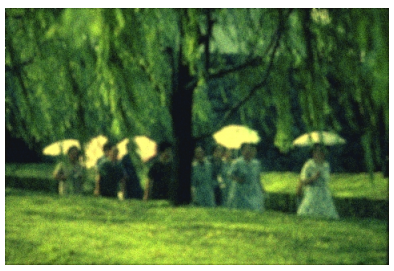

(e) stat. $-l \alpha \beta$

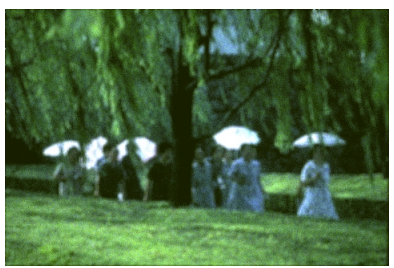

(i) stat. $-l u^{*} v^{*}$

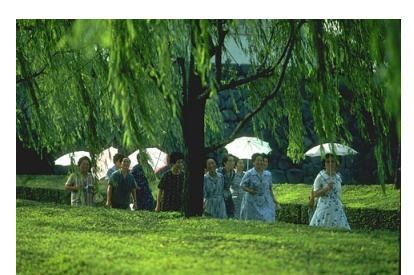

(b) Target image

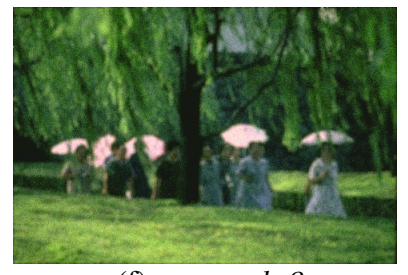

(f) geom. - $l \alpha \beta$

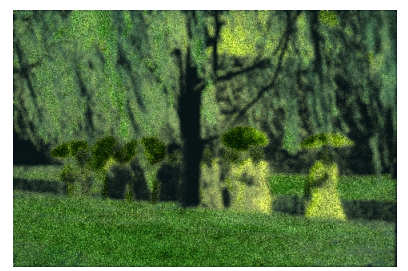

(j) geom. $-l u^{*} v^{*}$

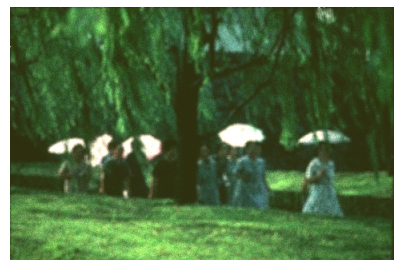

(c) stat. - RGB

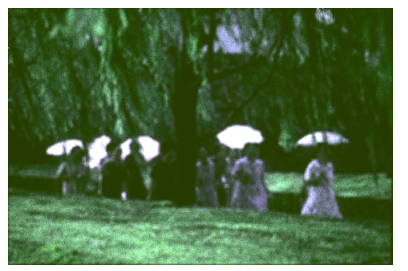

(g) stat. - $l a^{*} b^{*}$

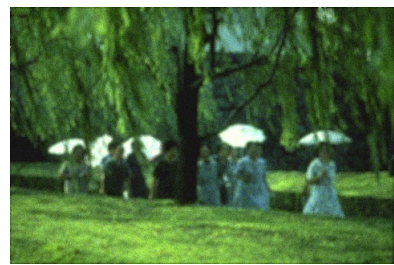

(k) stat. - RLAB

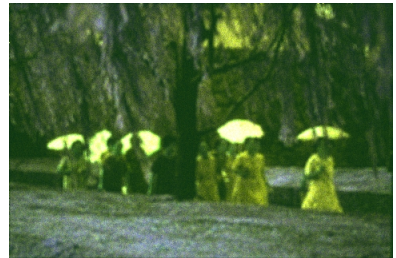

(d) geom. - RGB

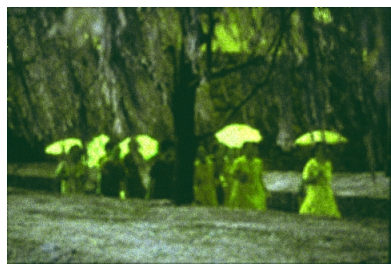

(h) geom. - $l a^{*} b^{*}$

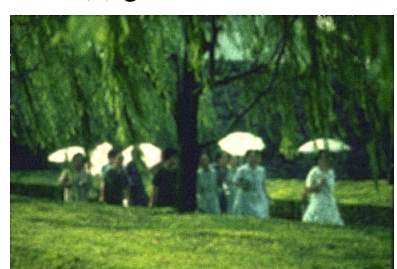

(1) geom. - RLAB

Figure 5: An example of color transfer using the BSD300 dataset: (a) a source image, (b) a target image, (c)-(1) results obtained using statistical and geometrical methods in the order of RGB, $l \alpha \beta, l a^{*} b^{*}, l u^{*} v^{*}$, and RLAB color spaces. Note that stat. $=$ statistical method, geom. $=$ geometrical method.

Table 3: Quantitative evaluation of the images in Fig. 5 by histogram matching

\begin{tabular}{|l|cc|cc|cc|cc|}
\hline \multirow{2}{*}{ Color space } & \multicolumn{2}{|c|}{ Euclidean } & \multicolumn{2}{|c|}{ Bhattacharyya } & \multicolumn{2}{c|}{ Chi-square } & \multicolumn{2}{c|}{ Intersection } \\
\cline { 2 - 9 } & stat. & geom. & stat. & geom. & stat. & geom. & stat. & geom. \\
\hline RGB & 0.4586 & 0.0913 & 1.0265 & 0.4327 & 1.6852 & 0.3794 & 0.9280 & 1.3670 \\
\hline$l \alpha \beta$ & 0.0905 & 0.0770 & 0.4341 & 0.3185 & 0.4285 & 0.1750 & 1.3445 & 1.5113 \\
\hline$l a^{*} b^{*}$ & 0.3148 & 0.1043 & 0.7657 & 0.4338 & 0.9338 & 0.3517 & 1.0066 & 1.3589 \\
\hline$l u^{*} v^{*}$ & 0.1058 & 0.0783 & 0.3904 & 0.3585 & 0.3202 & 0.2430 & 1.3552 & 1.4143 \\
\hline RLAB & 0.0700 & 0.0534 & 0.3152 & 0.2182 & 0.2084 & 0.1008 & 1.4574 & 1.5389 \\
\hline
\end{tabular}

The Italic number in a numerical cell indicates the best value of a metric. stat. $=$ statistical method; geom. $=$ geometrical method.

For the comparison of histogram, we use the equation

$$
d_{C}^{k}\left(H_{d}^{k}, H_{t}^{k}\right)=\sum_{i=0}^{b-1} \frac{\left(H_{d}^{k}(i)-H_{t}^{k}(i)\right)^{2}}{H_{d}^{k}(i)+H_{t}^{k}(i)},
$$

where chi-square distance is in the range $[0.0,2.0]$.

The last measurement is the intersection metric that is the intersection of the two histograms and is defined as

$$
d_{I}^{k}\left(H_{d}^{k}, H_{t}^{k}\right)=\sum_{i=0}^{b-1} \min \left(H_{d}^{k}(i), H_{t}^{k}(i)\right),
$$

where the intersection metric is in the range $[0.0,1.0]$.

Because a color image has three channels, a color histogram comprises three histograms calculated for each channel. For the comparison of color histograms, we combine the three metrics for each channel as a vector in threedimensional space. A comprehensive distance metric is then computed from the metric $d^{k}$ of each channel as a length of the vector using $d_{*}=\sqrt{\left(d_{*}^{1}\right)^{2}+\left(d_{*}^{2}\right)^{2}+\left(d_{*}^{3}\right)^{2}}$, where $*$ represents $E, B, C$, or $I$ for each metric. A smaller distance value indicates better similarity of histograms for Euclidean, Bhattacharyya, and chi-square metrics and thus the better color transfer. However, for the intersection metric, a higher value means better similarity.

\section{Experimental results}

Experiments were conducted on many images to compare the performance of the statistical method with that of the geometrical method in different color spaces. The two color transfer algorithms, written in C language, were implemented on a notebook computer running Windows 10 .

We used 100 natural dark color images from a dataset and 300 color images from the BSD300 dataset, with both datasets downloaded through the Internet[19]. For the sake of convenience, we refer to the former as dataset 1 and the 


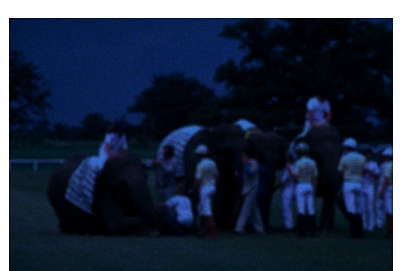

(a) Source image

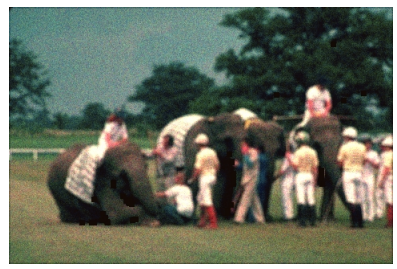

(e) stat. $-l \alpha \beta$

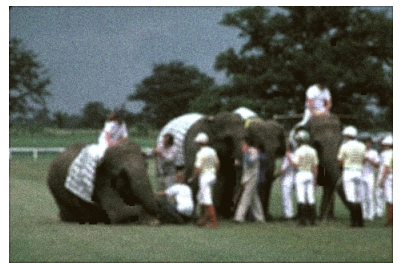

(i) stat. $-l u^{*} v^{*}$

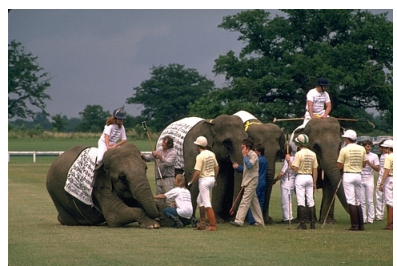

(b) Target image

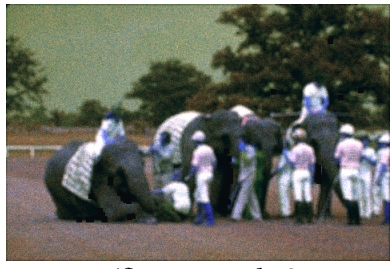

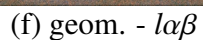

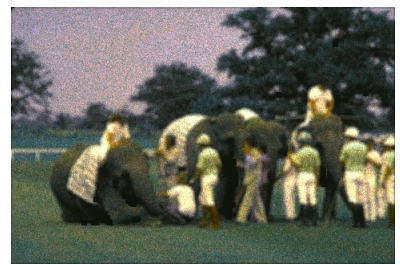

(j) geom. $-l u^{*} v^{*}$

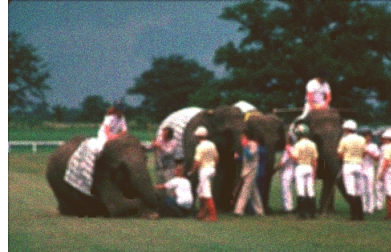

(c) stat. - RGB

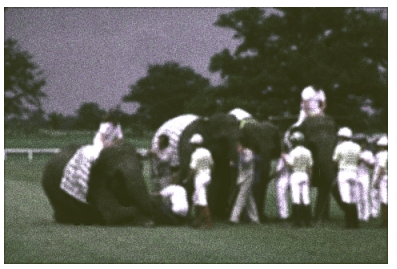

(g) stat. - $l a^{*} b^{*}$

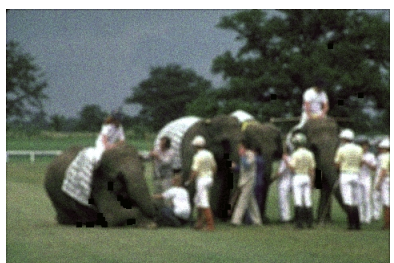

(k) stat. - RLAB

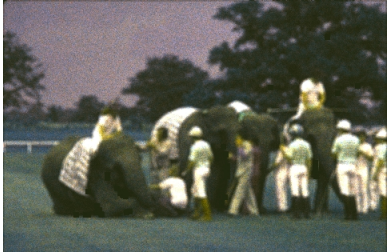

(d) geom. - RGB

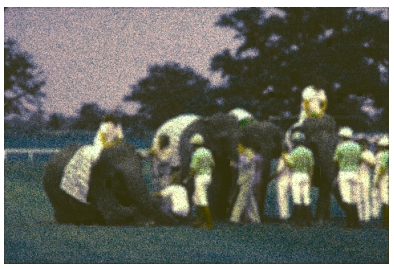

(h) geom. - $l a^{*} b^{*}$

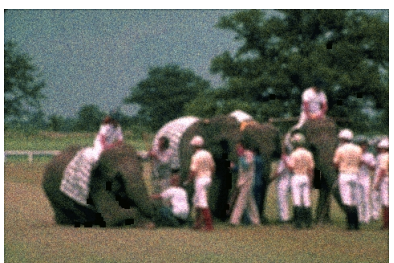

(1) geom. - RLAB

Figure 6: Another example of color transfer using the BSD300 dataset: (a) a source image, (b) a target image, (c)-(l) results obtained using statistical and geometrical methods in the order of RGB, $l \alpha \beta, l a^{*} b^{*}, l u^{*} v^{*}$, and RLAB color spaces. Note that stat. = statistical method, geom. = geometrical method.

Table 4: Averages of the quantitative evaluation for all BSD300 dark images by histogram matching

\begin{tabular}{|l|cc|cc|cc|cc|}
\hline \multirow{2}{*}{ Color space } & \multicolumn{2}{|c|}{ Euclidean } & \multicolumn{2}{c|}{ Bhattacharyya } & \multicolumn{2}{c|}{ Chi-square } & \multicolumn{2}{c|}{ Intersection } \\
\cline { 2 - 9 } & stat. & geom. & stat. & geom. & stat. & geom. & stat. & geom. \\
\hline RGB & 0.4739 & 0.1365 & 1.1110 & 0.5151 & 1.8495 & 0.4797 & 0.7692 & 1.2887 \\
\hline$l \alpha \beta$ & 0.1248 & $\mathbf{0 . 1 1 3 2}$ & 0.4359 & $\mathbf{0 . 4 0 4 5}$ & 0.3721 & $\mathbf{0 . 3 3 3 0}$ & 1.3591 & $\mathbf{1 . 3 8 2 7}$ \\
\hline$l a^{*} b^{*}$ & 0.2284 & 0.1646 & 0.7089 & 0.5663 & 0.8378 & 0.5996 & 1.0724 & 1.2098 \\
\hline$l u^{*} v^{*}$ & 0.1318 & 0.1217 & 0.4563 & 0.4233 & 0.3921 & 0.3757 & 1.3523 & 1.3391 \\
\hline RLAB & $\mathbf{0 . 1 0 7 5}$ & 0.1205 & $\mathbf{0 . 4 0 9 5}$ & 0.4378 & $\mathbf{0 . 3 2 6 6}$ & 0.3792 & $\mathbf{1 . 4 0 0 7}$ & 1.3456 \\
\hline
\end{tabular}

The Italic number in a numerical cell indicates the best value of a metric.

The bold number is the best value in a column.

stat. = statistical method; geom. $=$ geometrical method

later as dataset 2 . We used the dark color images in dataset 1 as source images and selected 10 natural images in dataset 2 as target images. We transferred color information from the 10 target images in the five color spaces using the two methods, resulting in 100 images for one dark image. Therefore, 10,000 images were produced.

Figure 3 presents experimental results obtained when using the two color transfer methods in different color spaces. Figure 3(a) shows a source image, which is an extremely dark image taken from dataset 1 . The intensity is so low that the content can hardly be observed by the human eye. Figure 3(b) shows a target image taken from dataset 2, in which the color information is transferred to the source image (a). Figure 3(c) and (d) respectively shows the results of direct transference in the RGB color space obtained using statistical and geometrical methods. Figure 3(e) and (f) respectively shows the results of color transference in the $l \alpha \beta$ color space obtained using statistical and geometrical methods. Likewise, Fig. $3(\mathrm{~g})$ and $(\mathrm{h})$ shows results for the $l a^{*} b^{*}$ space, Fig. 3(i) and (j) shows results for the $l u^{*} v^{*}$ space, and Fig. 3(k) and (l) shows results for the RLAB color space, with Fig. 3(g), (i), and (k) presenting the results for the statistical method and Fig. 3(h), (j), and (l) the results for the geometrical method.

Table 1 presents the results of quantitative evaluation using histogram matching metrics mentioned in the previous section. The first column gives the color spaces, the second to fifth columns give the Euclidean, Bhattacharyya, chisquare, and intersection metrics in that order. In the second row, stat. and geom. respectively refer to the statistical method and geometrical method. Each cell includes the evaluated numerical results. A larger value indicates a better result for the intersection metric while a smaller value indicates a better result for the other three metrics. The best 
value is given in italics. The table reveals that the geometrical method performs better than the statistical method in all color spaces for all evaluation metrics, except for the chisquare metric in the $l \alpha \beta$ color space. In this space, the geometrical method (2.7925) performs a little worse than the statistical method (2.7918) according to the chi-square metric. At first glance, the results of the statistical method seem better than those of the geometrical method owing to slight over-saturation of the sky. However, careful observation reveals that the colors of the lawn are more similar to those in the target image when using the geometrical method.

In this experiment, we obtained 10,000 resulting images and calculated the four metrics for each image and its corresponding target image. The geometrical method certainly outperforms the statistical method for most images, but not for all images. We therefore calculated the average of each metric in each color space for each color transfer method as listed in Table 2. The notations in this table are the same as those in Table 1, but the values in the cells are the average values for each metric. The italic number in a numerical cell indicates the best value of a metric, while the bold number indicates the best value in a column. By observing the data in each cell, we find that the geometrical method outperforms the statistical method for all color spaces and all metrics, except for the combination of the $l u^{*} v^{*}$ color space and Bhattacharyya metric. Note that, except for the combination of the Bhattacharyya metric and geometrical method, among the five color spaces, the RLAB color space has the best performance. This result is consistent with the conclusion drawn by Jonathan et al., who claimed that the RLAB color space is the best space for color transfer[9], although they only used the statistical method in their experiments.

In the next experiment, we used 300 images from dataset 2. All images had been converted to dark images; i.e., nighttime images[20]. We directly used the converted dark images downloaded[19]. We used the original image as the target image, and the converted dark image as the corresponding source image. Color information in the original images was transferred to the dark images. Each pair of source and target images provided 10 resulting images in five color spaces for the two color transfer methods, and we thus obtained 3000 resulting images.

Figure 5 presents experimental results for dataset 2 (i.e., the BSD300 dataset). Figure 5(a) shows a dark image created from the original in Fig. 5(b) to simulate a night scene. The dark image was used as the source image, while the original image was used as the target image, in which the color was transferred to the source image. Figure 5(c) and (d) respectively show resulting images obtained using the statistical method and geometrical method in the RGB color space. Similarly, Fig. 5(e) and (f), (g) and (h), (i) and (j), and $(\mathrm{k})$ and (l) respectively show images produced in $l \alpha \beta$, $l a^{*} b^{*}, l u^{*} v^{*}$, and RLAB color spaces using the two methods.

Table 3 presents the quantitative evaluation of images in Fig. 5 by histogram matching. The notations are the same as those in Tables 1 and 2 . The table shows that the geometrical method outperforms the statistical method for all metrics in all color spaces.
We transferred color information in original images to the 300 artificially created dark images individually to obtain 3000 images as described above. One result is shown in figure 6, where Fig. 6(a) shows the dark image created from the original image in Fig. 6(b) to simulate a night scene. For the transference of color information of the image in Fig. 6(b) to the image in Fig. 6(a) using statistical and geometrical methods, the results are shown in Fig. 6(c) and (d) for the RGB color space, Fig. 6(e) and (f) for the $l \alpha \beta$ color space, Fig. $6(\mathrm{~g})$ and $(\mathrm{h})$ for the $l a^{*} b^{*}$ color space, Fig. 6(i) and (j) for the $l u^{*} v^{*}$ color space, and Fig. 6(k) and (l) for the RLAB color space. As before, the four metrics were calculated for each histogram pair of the original image and resulting image. Average values of the metrics are presented in Table 4. This quantitative evaluation reveals that the geometrical method outperforms the statistical method for all metrics and color spaces except for the RLAB space. When applying the statistical method for color transfer, the RLAB color space had the best performance according to all metrics. This is again consistent with the opinion of Jonathon et al. The next best color space when using the statistical method was the $l \alpha \beta$ space. However, for geometrical method, as shown in bold numbers, the $l \alpha \beta$ space provided the best performance among color spaces.

The following finds are taken from the above experiments. The geometrical method generally outperforms the statistical method in most color spaces according to the histogram matching metrics in both cases of natural dark images and artificially created night scenes. When transferring color information to a dark image using the statistical method, the RLAB space is generally the best color space, although not in all cases. When using the geometrical method for color transference on the BSD300 dataset, the $l \alpha \beta$ space is a better color space in most cases. We recognize that there is no best color space and color transfer method for all situations.

\section{Conclusions}

In this comparative study on statistical and geometrical methods of transferring color information from a given original image to a dark image, we adopted four commonly used color spaces and a special RLAB color space to conduct color transfer and used four histogram matching metrics to evaluate the methods and color spaces. Through quantitative comparison, we found that the geometrical method outperforms the statistical method in most cases. Generally speaking, the RLAB space is the best color space for color transfer, seemingly followed by the $l \alpha \beta$ space. It is clear that the best color space and method depends on the specific contents of source and target images. We are still addressing what dependencies should be considered. Color transfer in the present study is only from image to image, and we will evaluate color transfer from swatch to swatch in future work.

\section{Acknowledgment}

This work is part of research supported by the Nature Science Foundation of Qinghai Province under Grant No. 
2016-ZJ-745, the Project of the Chongqing Nature Science Foundation under Grant No. cstc2019jcyj-msxmX0683, and the National Nature Science Foundation of China under Grant Nos. 61662062 and 61602088.

\section{References}

[1] H. S. Faridul, T. Pouli, C. Chamaret, J. Stauder, A. Tremeau, and E. Reinhard: "A Survey of Color Mapping and its Applications", In Proc. of Eurographics 2014 - State of the Art Reports (2014).

[2] F. Pitie and A. Kokaram: "The Linear Monge-Kantorovitch Linear Colour Mapping for Example-based Colour Transfer", In IEE European Conference on Visual Media Production (CVMP'07)(2007).

[3] L. A. Gatys, A. S. Ecker, and M. Bethge: "Image Style Transfer Using Convolutional Networks", in IEEE Conference on Computer Vision and Pattern Recognition (CVPR2016)(2016)

[4] L. A. Gatys, M. Bethge, A. Hertzmann, and E. Shechtman: "Preserving Color in Neural Artistic Style Transfer", arXiv, http://arxiv.org/abs/1606.05897(2016).

[5] E. Reinhard, M. Ashikhmin, B. Gooch, and P. Shirley: "Color Transfer between Images", IEEE Computer Graphics and Applications", 21(5), 34-41(2001).

[6] E. Reinhard and T. Pouli: "Colour Spaces for Colour Transfer”, In: Schettini R., Tominaga S., Trémeau A. (eds) Computational Color Imaging. CCIW 2011. Lecture Notes in Computer Science, Vol. 6626. Springer, Berlin, Heidelberg(2011).

[7] X. Xiao and L. Ma: "Color Transfer in Correlated Color Space", In Proc. of the 2006 ACM Intl. Conf. on Virtual Reality Continuum and its Applications, ACM, 305-309, (2006).

[8] M. Fairchild: "Refinement of the RLAB Color Space", Color Research and Application, 21(5), 338-346, (1996).

[9] C. N. Jonathan, E. S. Y. Raul, E. C. T. Fernando, and A. L. M. Rocio: "Dark Image Enhancement Using Perceptual Color Transfer", IEEE Access, 6, 4935-14945, (2017).

[10] A. Bhattacharyya, "On a Measure of Divergence between Two Statistical Populations Defined by their Probability Distribution”, Bull. Calcutta Math. Soc., 35(1), 99-109, (1943).

[11] https://en.wikipedia.org/wiki/Bhattacharyya_ distance, Accessed on Jan. 2020.

[12] N.D. Gagunashvili: "Chi-square Tests for Comparing Weighted Histograms", arXiv:0905.4221 (2009).

[13] H. S. Faridul, T. Pouli, C. Chamaret, J. Stauder, D. Kuzovkin, A. Tremeau, and E. Reinhard: "Color Mapping: A Review of Recent Methods, Extensions, and Applications", Computer Graphics Forum, 35(1) (2016).

[14] R. C. Gonzalez, and R. E. Woods, [Digital Image Processing], Fourth Ed., pp.530-537, Pearson Prentice Hall, New Jersey (2018).
[15] D. L. Ruderman, T. W. Cronin, and C. C. Chiao: "Statistics of Cone Response to Natural Images: Implications for Visual Coding", Journal of the Optical Society of America A, 15(8), 2036-4045 (1998)

[16] X. Zhang, W. Jiang, X. Liu, and X. Lei: "Visualization of Color Transfer between Images in RGB Color Space", International Workshop on Advanced Image Technology (IWAIT2018), P2V, Chiang Mai, Thailand (2018).

[17] W. H. Press, S. A. Teukolsky, W. T. Vetterling, and B. P. Flannery: Numerical recipes in $\mathrm{C}++$ The art of scientific computing (2nd Ed.), Publishing House of Electronics Industry, 68-73 (2003) (in Chinese).

[18] Y. Rubner, C. Tomasi, and L. J. Guibas, "A Metric for Distributions with Applications to Image Databases", International Journal of Computer Vision, 40(2), 99-121 (2000).

[19] https://jonathancn16.wixsite.com/profile/ publications, Accessed on Jan. 2020.

[20] W. B. Thompson, P. Shirley, and J. A. Ferwerda, "A Spatial Post-processing Algorithm for Images of Night Scene", Journal of Graphic Tools, 7(1), 1-12 (2002).

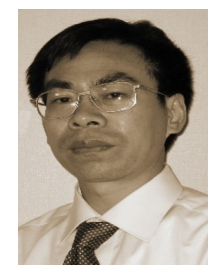

Xiaohua Zhang (Member) received a $\mathrm{PhD}$ degree in information science and engineering from the Tokyo Institute of Technology, Japan, in 2000. After working at NHK Engineering System Inc. from 2000 to 2003 , he joined the faculty of the Hiroshima Institute of Technology as a professor. His research interests include computer graphics, image processing, computer vision, pattern recognition, and machine learning. He is a member of the IEEE, IEICE, ITE, IIEEJ, and IIAE.

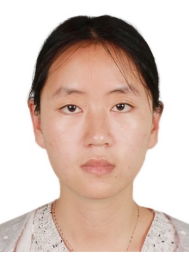

Dongdong Cheng (Non-member) received a B.S. degree in computer science from Chongqing Normal University, China, in 2013, and a PhD degree from Chongqing University, China, in 2018. She is now a lecturer at the College of Big Data and Intelligent Engineering at Yangtze Normal University. Her research interests are clustering analysis and data mining.

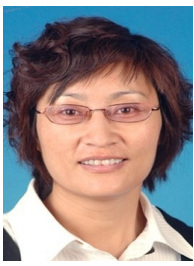

Yuelan Xin (Non-member) received a B.S. degree in physics from Shaanxi Normal University, China, in 1997, an M.S. degree in computer science and engineering from Qinghai Normal University, China, in 2009, and a $\mathrm{PhD}$ degree from Shaanxi Normal University, China, in 2018. She is currently a professor at Qinghai Normal University. Her research interests include image processing and pattern recognition. 